\title{
Identification of PLA2G7 as a novel biomarker of diffuse large B cell lymphoma
}

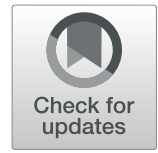

Weili Zheng ${ }^{1 \dagger}$, Qiaochu Lin ${ }^{1 \dagger}$, Mohammed Awal Issah', Ziyuan Liao ${ }^{2}$ and Jianzhen Shen ${ }^{1 *}$

\begin{abstract}
Background: Diffuse large B-cell lymphoma is the most common form of non-Hodgkin lymphoma globally, and patients with relapsed or refractory DLBCL typically experience poor long-term outcomes.

Methods: Differentially expressed genes associated with $D L B C L$ were identified using two GEO datasets in an effort to detect novel diagnostic or prognostic biomarkers of this cancer type, after which receiver operating characteristic curve analyses were conducted. Genes associated with DLBCL patient prognosis were additionally identified via WCGNA analyses of the TCGA database. The expression of PLA2G7 in DLBCL patient clinical samples was further assessed, and the functional role of this gene in DLBCL was assessed through in vitro and bioinformatics analyses.

Results: DLBCL-related DEGs were found to be most closely associated with immune responses, cell proliferation, and angiogenesis. WCGNA analyses revealed that PLA2G7 exhibited prognostic value in DLBCL patients, and the upregulation of this gene in DLBCL patient samples was subsequently validated. PLA2G7 was also found to be closely linked to tumor microenvironmental composition such that $D L B C L$ patients expressing higher levels of this gene exhibited high local monocyte and gamma delta $T$ cell levels. In vitro experiments also revealed that knocking down PLA2G7 expression was sufficient to impair the migration and proliferation of DLBCL cells while promoting their apoptotic death. Furthmore, the specific inhibitor of PLA2G7, darapladib, could noticeably restrained the DLBCL cell viability and induced apoptosis.
\end{abstract}

Conclusions: PLA2G7 may represent an important diagnostic, prognostic, or therapeutic biomarker in patients with DLBCL.

Keywords: Diffuse large B-cell lymphoma, Receiver operating characteristic curve, Weighted gene co-expression network analysis, Tumor microenvironment, PLA2G7

\section{Introduction}

Lymphomas are a very prevalent form of cancer that can be classified into Hodgkin and non-Hodgkin lymphoma subtypes (HL and NHL, respectively). Diffuse large Bcell lymphoma (DLBCL) is the most common NHL subtype globally, accounting for $30-40 \%$ of overall NHL cases [1]. DLBCL is a highly heterogeneous and

\footnotetext{
* Correspondence: shenjianzhen@fjmu.edu.cn

'Weili Zheng and Qiaochu Lin contributed equally to this work.

${ }^{1}$ Fujian Institute of Hematology, Fujian Medical Center of Hematology, Fujian

Provincial Key Laboratory on Hematology; Fujian Medical University Union Hospital, Fuzhou, China

Full list of author information is available at the end of the article
}

aggressive disease that can exhibit highly varied outcomes in affected patients. Treatment of DLBCL patients with rituximab and cyclophosphamide-doxorubicinvincristine-prednisone chemotherapy ( $\mathrm{R}-\mathrm{CHOP})$ has led to rising long-term patient survival and a $50-75 \% 5$-year survival rate for patients with this disease [2]. However, roughly $40 \%$ of patients ultimately suffer from relapsed or refractory disease [3]. The genetic and molecular etiology of DLBCL is also highly complex and some studies have focused on the identification of genetic drivers and their functional roles in DLBCL to determine novel therapeutic targets and/or diagnostic or prognostic

(c) The Author(s). 2021 Open Access This article is licensed under a Creative Commons Attribution 4.0 International License, which permits use, sharing, adaptation, distribution and reproduction in any medium or format, as long as you give appropriate credit to the original author(s) and the source, provide a link to the Creative Commons licence, and indicate if changes were made. The images or other third party material in this article are included in the article's Creative Commons licence, unless indicated otherwise in a credit line to the material. If material is not included in the article's Creative Commons licence and your intended use is not permitted by statutory regulation or exceeds the permitted use, you will need to obtain permission directly from the copyright holder. To view a copy of this licence, visit http://creativecommons.org/licenses/by/4.0/ The Creative Commons Public Domain Dedication waiver (http://creativecommons.org/publicdomain/zero/1.0/) applies to the data made available in this article, unless otherwise stated in a credit line to the data. 
biomarkers to promote the disease's diagnosis and treatment $[4,5]$.

High-throughput and next-generation sequencing (NGS) technologies have enabled many biomarker discovery studies in DLBCL samples to date [6,7]. Findings from the majority of these studies, however, have yet to be validated or implemented in clinical settings. Many of these prior studies have also been limited by factors such as tissue heterogeneity, small sample sizes, and singlecohort approaches, resulting in inconsistent results [8]. One approach to overcoming these limitations is the reanalysis of multiple independent previously published NGS datasets in order to more reliably identify potential cancer-specific biomarkers $[9,10]$.

Herein, we sought to identify novel DLBCL diagnostic, prognostic, or therapeutic biomarkers via an integrative bioinformatics approach. Through this strategy, we identified phospholipase A2 group VII (PLA2G7) as a novel biomarker of interest associated with this cancer type. PLA2G7 has previously been reported to control a range of oncogenic processes through mechanisms associated with metabolic alterations [11]. PLA2G7 has also been identified as a prognostic biomarker in patients with prostate cancer ( $\mathrm{PCa}$ ) [12] and melanoma [13]. Together, our data suggest that PLA2G7 may function as a driver of the proliferation, migration, and survival of tumor cells and a regulator of immune cell infiltration within the DLBCL tumor microenvironment. PLA2G7 may therefore represent a viable therapeutic target in DLBCL patients.

\section{Materials and methods}

\section{Ethics statement and clinical specimens}

The study was approved by the Ethics Committee of $\mathrm{Fu}$ jian Medical University Union Hospital. All experiments were performed according to the relevant regulations and written informed consent was obtained from patients. A total of 18 DLBCL tissues, 11 lymphadenitis tissues and 53 DLBCL peripheral blood samples were obtained from Fujian Medical University Union Hospital. Tissue samples were frozen in liquid nitrogen until further analysis. DLBCL cases only were confirmed by pathological examination of lymph node biopsy or lymphadenectomy according to the 2017 WHO Classification of Lymphoid Neoplasms. Patients samples used for this study had not received chemotherapy or radiotherapy before surgery.

\section{DEG identification}

The GSE32018 and GSE56315 datasets from the Gene Expression Omnibus (GEO) database (http://www.ncbi. nlm.nih.gov/geo/) were downloaded. Raw CEL files were background corrected, subjected to z-score transformation and quantile normalization, and subjected to further analysis. The GSE32018 dataset [14] contained 22 DLBCL tumor samples and 7 normal lymph node tissue samples and was prepared using the GPL6480 platform. The GSE56315 dataset [15] contained 55 DLBCL tissue samples and 33 normal tonsil tissues and was prepared using the GPL570 platform.

In addition, gene expression profiles and clinical data pertaining to 48 DLBCL patients were downloaded from The Cancer Genome Atlas (TCGA) database (https:// portal.gdc.cancer.gov/). The ESTIMATE algorithm was used to calculate stromal and immune scores [16].

Genes that were differentially expressed between DLBCL and control tissues were identified with the $R$ 'limma' package using the following cutoff criteria: $\log 2$ FoldChange $(\mathrm{FC})>1$ and adjusted $P$-value $<0.05$. The RobustRankAggreg (RRA) R package was then used to integrate the DEGs from these two datasets. RRA utilizes a probabilistic model to aggregate and monitor the genes that are ranked consistently better than expected under the null hypothesis of uncorrelated inputs, and defines a significance score for each gene [17]. Cutoff criteria: score $<0.05$ was applied.

\section{Functional enrichment analysis}

Identified DEGs were subjected to Gene Ontology (GO) and Kyoto Encyclopedia of Genes and Genomes (KEGG) pathway enrichment analyses [18-20] using the DAVID database (https://david.ncifcrf.gov/summary.jsp), with $P<0.05$ as the threshold of statistical significance.

Weighted gene co-expression network analysis (WGCNA) Co-expression analyses were conducted using the WGCNA R package using TCGA data corresponding to 48 DLBCL patients for whom detailed survival data were available. A weighted adjacency matrix was constructed by determining Pearson's correlation coefficients for individual pairs of genes. An appropriate soft power threshold was then selected to yield standardized scalefree networks, after which the resultant matrix was subjected to transformation into a topological overlap matrix (TOM). In addition, topological overlap dissimilarity (1-TOM) was utilized as input for hierarchical clustering analyses. Gene dendrogram and module identification were constructed with a dynamic tree cut. Any modules with a size $<30$ were then deleted. Modules that had a dissimilarity of $<0.25$ were merged, after which relationships between clinical traits and module eigengenes were assessed.

\section{Cell culture and transfection}

Human DB and SU-DHL-2 DLBCL cells were obtained from Procell Life Science and Technology and were grown in RPMI-1640 (Invitrogen, CA, USA) containing $10 \%$ fetal bovine serum (Gibco, CA, USA) at $37^{\circ} \mathrm{C}$ in a 
5\% CO2 humidified incubator. Cells were transfected with Negative Control (NC) and PLA2G7 siRNAs using Lipofectamine 3000 (Invitrogen). The siRNA sequences were as follows: PLA2G7-si1: CCUGUUGCCCAUAU GAAAUTT,

AUGGUUAAUGUUUGCAGGCAT; PLA2G7-si2: CCUGGAUGCAUGGAUGUUUTT, AAACAUCCAUGCAUCCAGGGC.

\section{Quantitative real-time PCR}

Trizol (Invitrogen) was utilized to extract RNA from cells, after which a cDNA synthesis kit (Thermo Fisher Scientific, USA) was used based on provided directions to prepared cDNA. The expression levels were detected by QRT-PCR analysis with FastStart Universal SYBRGreen Master (Roche), an ABI7500 sequence detector (Applied Biosystems, Foster City, CA, USA) and calculated by $2-\Delta \Delta C t$ method. $\beta$-actin expression was used for normalization purposes, and primers used in this study were as follows: PLA2G7, Forward (F): 5' -GAAC ACACTGGCTTATGGGC-3', Reverse (R): 5' -GAGATG CCAGGTCAATGCCA-3'.

\section{Colony formation and migration assays}

For colony formation assays, 500 cells were added per well in methylcellulose-based media. Plates were then cultured for 2 weeks, after which methanol was used to fix colonies which were subsequently stained with $0.5 \%$ crystal violet. All colonies containing $>50$ cells were then counted via microscopy.

For migration assays, 24-well transwell chambers ( $8 \mu \mathrm{m}$ aperture, BD Biosciences) were utilized. DLBCL cells were suspended in FBS-free media and were added to the upper well of these chambers, whereas cells in media supplemented with 10\% FBS were added into the lower chamber. Following a $48 \mathrm{~h}$ incubation, cells that had not migrated to the lower chamber were removed with a cotton swab. Migrated cells were then fixed and stained using $0.5 \%$ crystal violet and imaged via microscopy. In total, five fields of view per sample were analyzed at random. Image J was utilized to quantify the number of migrated cells per well.

\section{Apoptosis assay}

A PE Annexin V Apoptosis detection kit (BD Pharmingen, USA) was utilized at $48 \mathrm{~h}$ post-transfection to analyze cells. Briefly, cells were stained using Annexin V-PE and 7-AAD for $15 \mathrm{~min}$ at room temperature. A BD Accuri C6 flow cytometer was then used to analyze samples, after which FlowJo was used for data analysis.

\section{CCK-8 assay}

Cells were seeded into 96-well plates and incubated in different concentrations of darapladib for $72 \mathrm{~h}$, then
CCK- 8 dye solution was added to each well and incubated at $37^{\circ} \mathrm{C}$ for $2 \mathrm{~h}$. The absorbance was measured at $450 \mathrm{~nm}$ using a microplate reader (BioTek, USA).

\section{Statistical analysis}

ROC (receiver operating characteristic) curve was conducted to analyze the effectiveness of target gene expressions between tumor and healthy samples. Area under the ROC curve (AUC) values were calculated using SPSS 20.0 (SPSS Inc., IL, U.S.A.) and were used to assess the sensitivity and specificity of individual DEGs as diagnostic biomarkers between DLBCL and normal tissues.

Patients were separated into two groups based upon PLA2G7 expression levels, after which two-tailed chisquared tests were utilized to compare clinicopathological characteristics between the PLA2G7-high and -low patient groups. In addition, comparisons of PLA2G7 expression levels were made via Student's t-tests and oneway ANOVAs using GraphPad Prism 8. Kaplan-Meier survival curves were drawn to show the relationship between expression of PLA2G7 and overall survival (OS) of patients, which was tested by the log-rank test. $P<$ 0.05 was the significance threshold for this analysis.

\section{Results}

\section{Integrative DEG identification}

The general strategy for the present study is shown in Fig. 1. In total, 208 and 43 up- and down-regulated DLBCL-related DEGs, respectively, were identified in the GSE32018 dataset, while 993 and 1127 up- and downregulated DEGs, respectively, were identified in the GSE56315 dataset (Fig. 2A). These two datasets were then integrated via the RRA method, revealing 30 and 38 shared DEGs that were up- and down-regulated in DLBCL samples relative to normal tissue samples, respectively (Fig. 2B). KEGG enrichment analysis of these DEGs revealed that they were associated with the cell cycle, the NF-kB signaling pathway, and chemokine signaling (Fig. 2C). GO term enrichment analyses further revealed these DEGs to be associated with immune responses, the regulation of angiogenesis, cell proliferation, chemokine-mediated signaling, and cellular responses to tumor necrosis factor (Fig. 2D).

\section{Detection of potential diagnostic biomarkers of DLBCL}

ROC analyses were subsequently conducted for each of the 68 DEGs identified above, AUC values were used to assess the diagnostic sensitivity and specificity of these genes between DLBCL and normal tissues in the GSE56315 datasets. The top 20 more predictive of these DEGs, which yielded AUC values $>0.996$, are shown in Fig. 3A. In order to validate the diagnostic value of these 20 DEGs, these same ROC analyses were repeated using the GSE32018 dataset (Fig. 3B). A subset of these DEGs 


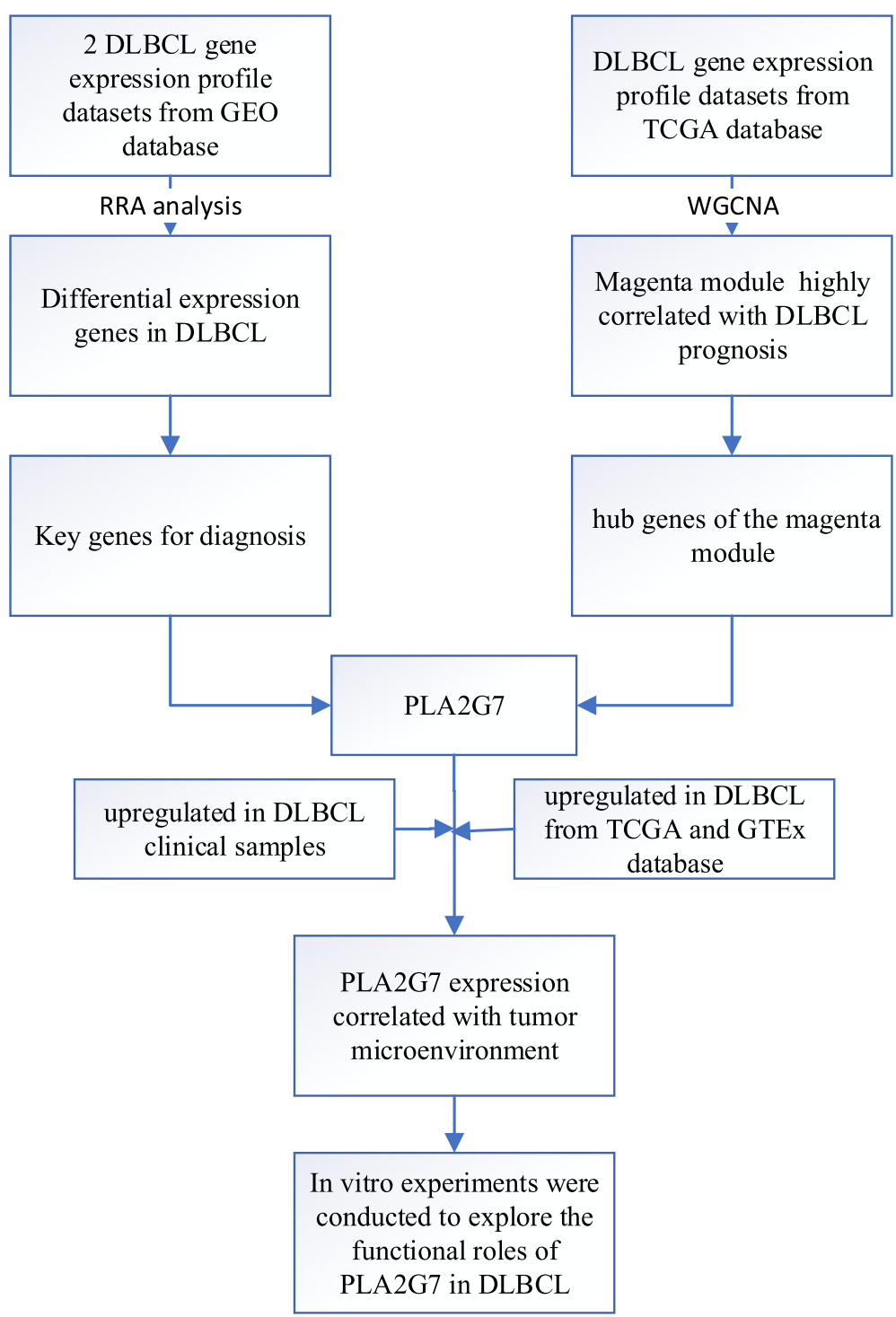

Fig. 1 Study overview

including KLHL23, FAP, PLA2G7, POSTN, and GPNMB, exhibited high AUC values, suggesting that they may be of significant value as diagnostic biomarkers of DLBCL (Fig. 3C-D).

\section{WGCNA and identification of key modules}

We next employed a systematic WGCNA approach to the unsupervised classification of these data, classifying DEGs into modules based upon their expression patterns [21]. Genes that exhibit highly correlated expression patterns are grouped into the same modules, as they are predicted to have identical or closely related biological functions, allowing for the direct assessment of the functional relevance of individual modules [22].
We conducted this WGCNA analysis based upon gene expression and survival data from 48 DLBCL patients in the TCGA database (Fig. 4A), and a scale-free network was constructed using a power of $\beta=5$ as a softthreshold (Fig. 4B). This approach ultimately grouped DEGs into 15 different modules that were identified and assigned different colors using a merged dynamic tree cut (Fig. 4C). The gray-colored module contained all genes that were not clustered with one another, and these genes were not analyzed further in this study. Correlations between module eigengene values and sample clinical traits are shown in Fig. 4D. The magenta module was found to be most closely associated with DLBCL patient prognosis, although this association was not 


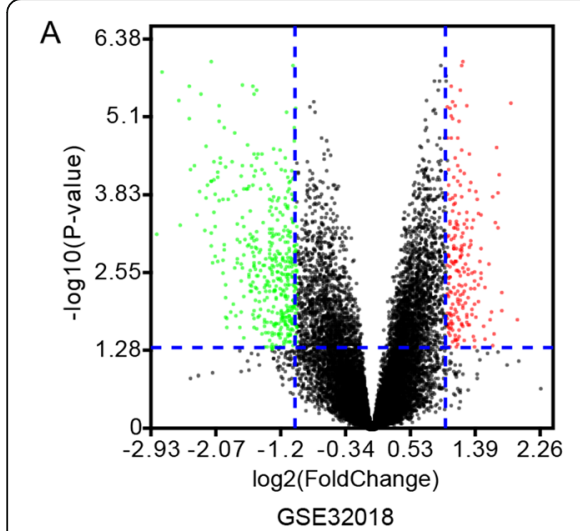

B

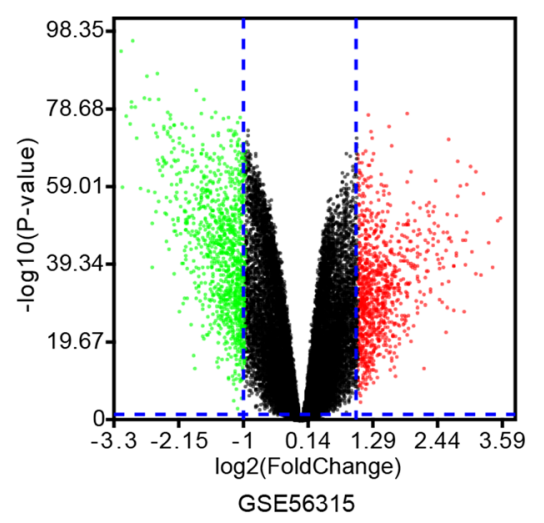

C

KEGG Pathway

D
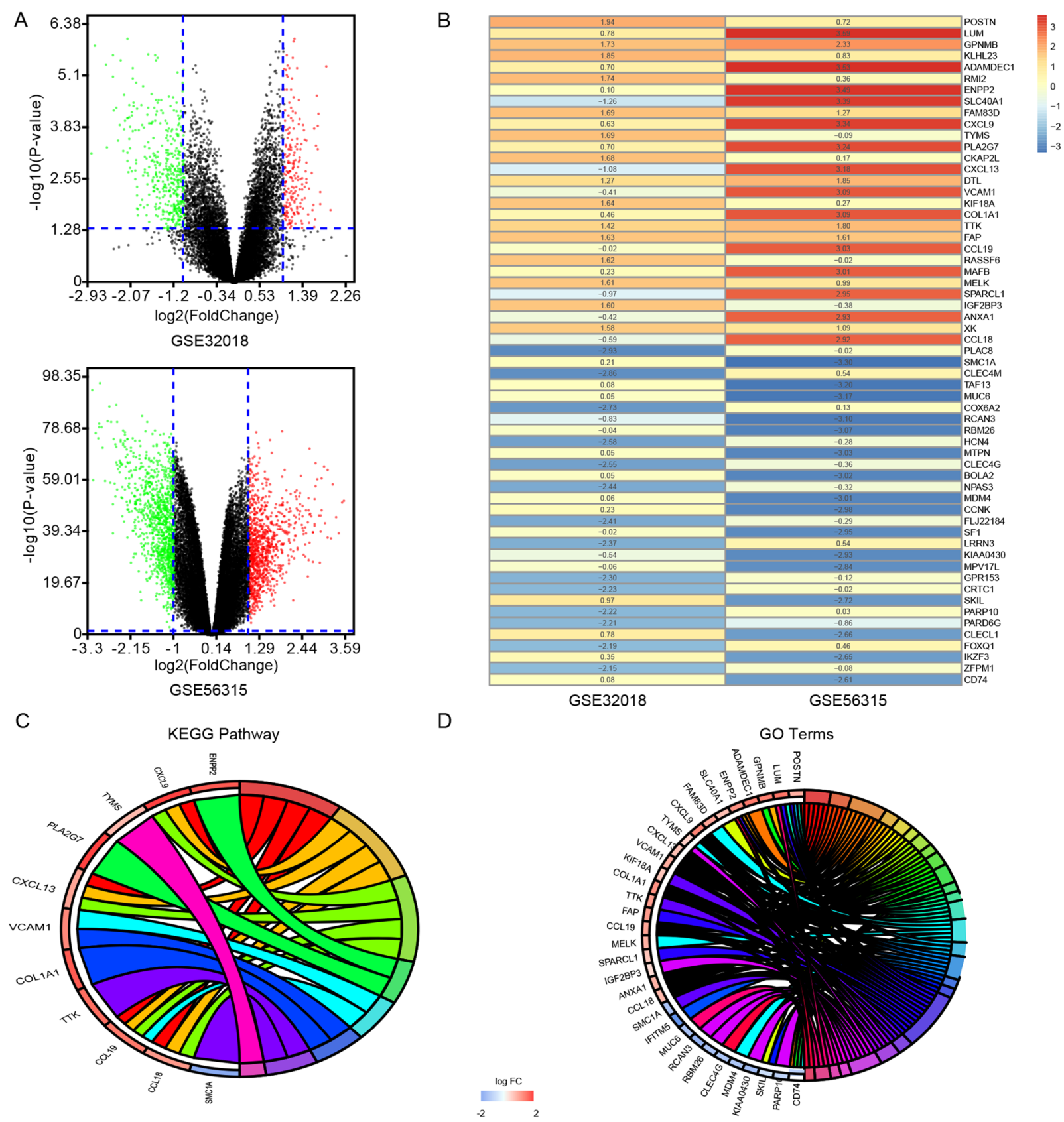

GSE32018

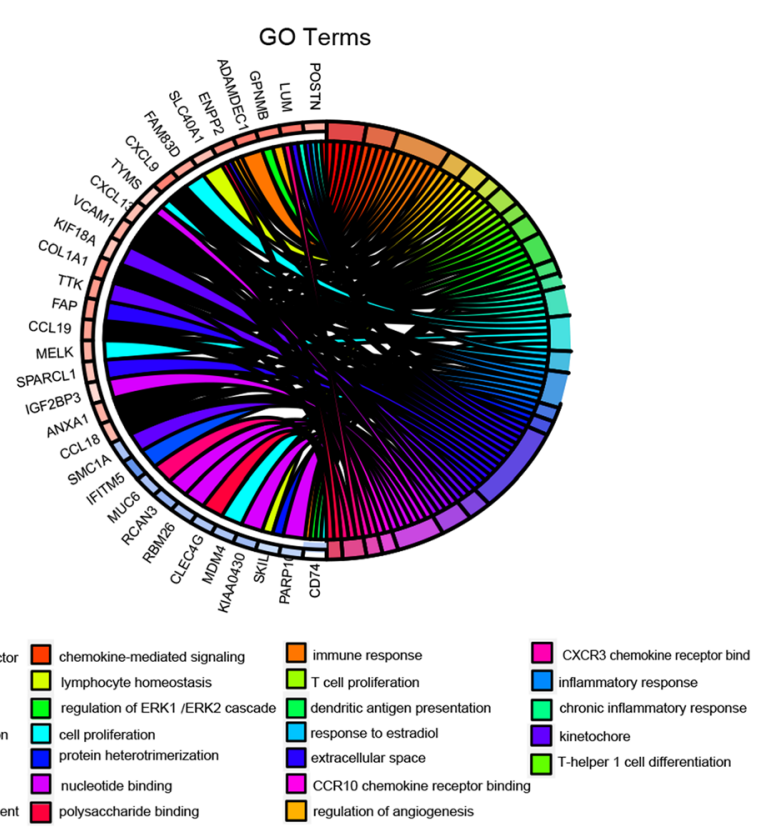

Fig. 2 DLBCL-related differentially expressed gene identification. A: Volcano plots were used to compare gene expression profiles in the GSE32018 and GSE56315 datasets. Red and green symbols are used to indicate genes that were significantly up- and down-regulated, respectively, as per the following criteria: $\log 2 \mathrm{FC}>1$ and adjusted $P$-value $<0.05$. B: The top 30 upregulated and top 38 downregulated genes in these two datasets were ranked using the Robust Rank Aggreg (RRA) approach. Individual rows corresponding to genes, while columns correspond to datasets. Red and blue represent genes that were up- and down-regulated, respectively, relative to normal tissues. C: DEGs were subjected to a KEGG pathway enrichment analysis. D: DEGs were subjected to GO term enrichment analyses 


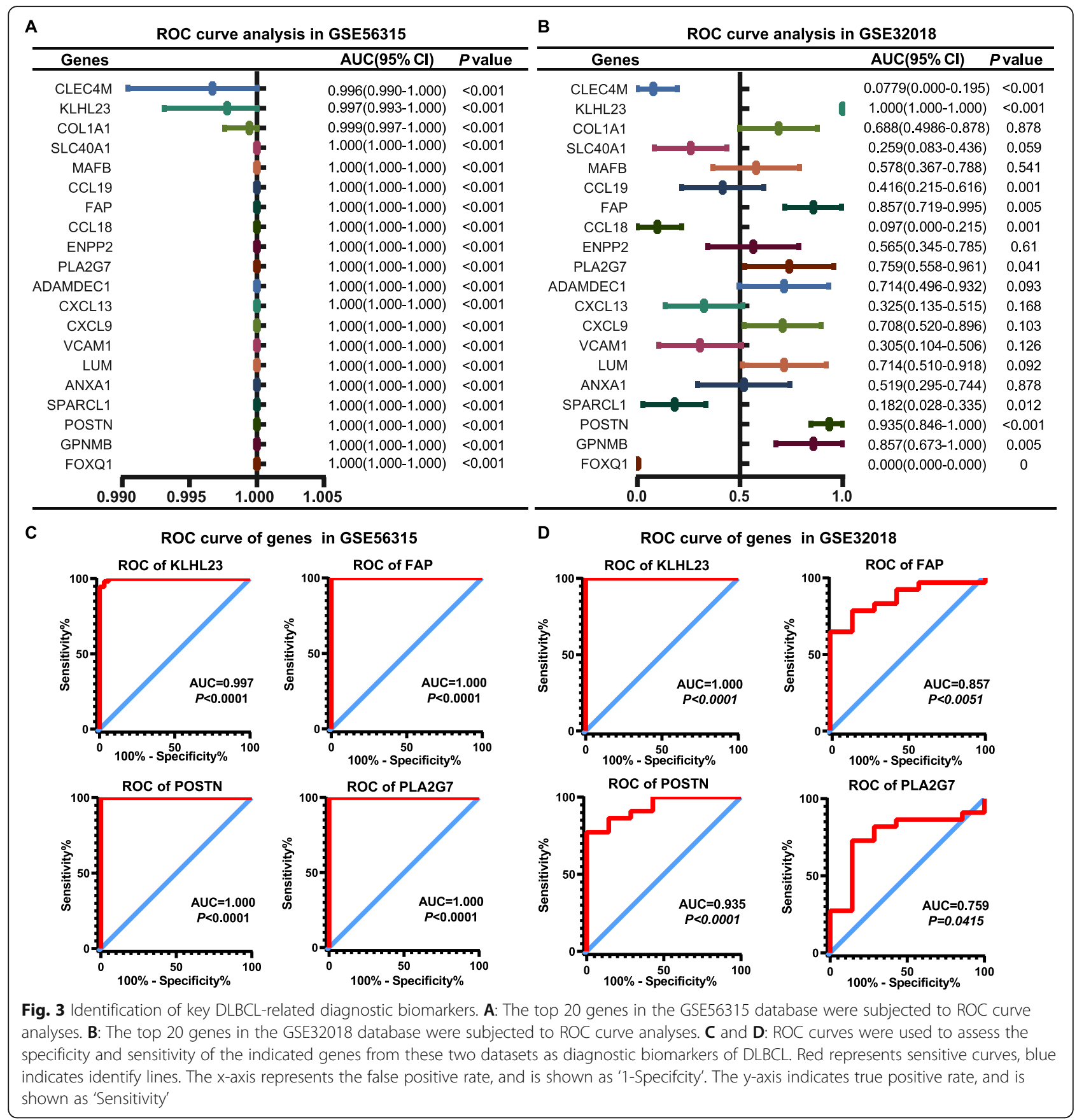

significant. $\left(R^{2}=0.26, P=0.07\right.$ with survival time; $R^{2}=$ $-0.2, P=0.2$ with survival status). Notably, this magenta module included the PLA2G7 gene, which we had identified as a promising diagnostic biomarker in our above analyses. Indeed, PLA2G7 was a hub gene within this magenta module $(\mathrm{MM}=0.84$ and $\mathrm{GS}=0.17$, genes in magenta module are shown in Supplemental Table S1), and it has previously been shown to function as a key regulator of oncogenesis and inflammation [11]. The expression and functionality of PLA2G7 in DLBCL, however, remains to be fully clarified.
Elevated PLA2G7 expression is linked to the DLBCL tumor microenvironment

To explore the mechanistic role of PLA2G7 in the context of tumor progression, we next conducted a GEPIAbased conjoint analysis of 33 tumor types within the TCGA and GTEx databases [23]. These analyses revealed that PLA2G7 expression was significantly elevated in 17 tumor types, including DLBCL, relative to corresponding normal tissue controls (Fig. 5A). We then conducted a qRT-PCR-based comparison of PLA2G7 mRNA expression in DLBCL patient tumor tissues and 


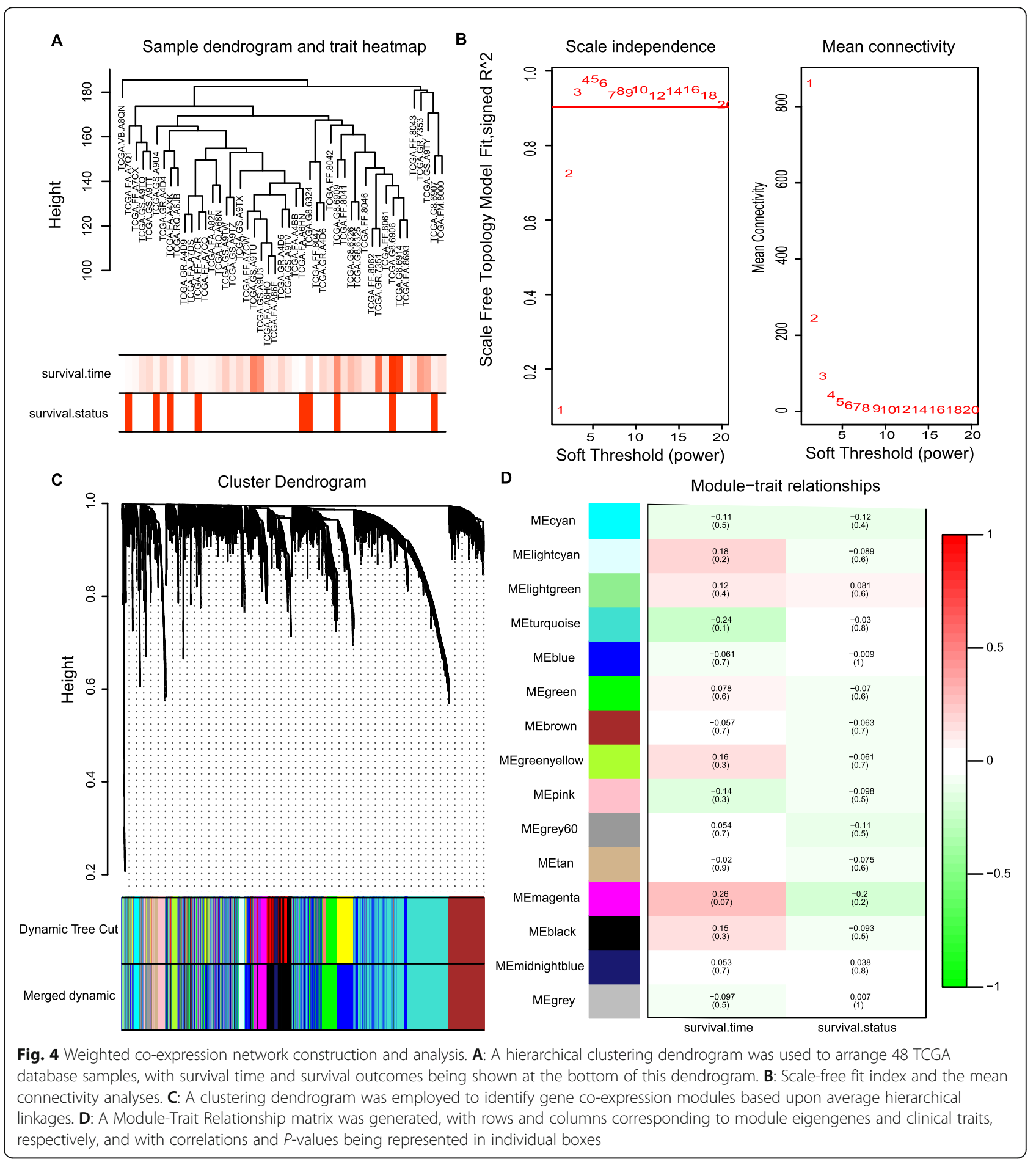

benign lymphadenitis patient tissues, leading us to confirm that this gene is highly expressed in DLBCL tissues (Fig. 5B, mean $=14.95$, 95\% confidence interval $=4.661-$ 25.23). Clinicopathological features in DLBCL patients were shown in Supplemental Table S2. Further analyses of the TCGA database revealed that high PLA2G7 expression (greater than the median value (cut-off $=10.32$ ) was associated with higher stromal $(P=0.021)$ and immune $(P=0.004)$ scores (Fig. $5 C-D)$. In contrast, these expression levels were not correlated with tumor size or stage (Table 1). These findings suggest that PLA2G7 expression is associated with intratumoral heterogeneity and the composition of the tumor microenvironment (TME). The TME consists of stromal cells, tumor cells, immune 
A
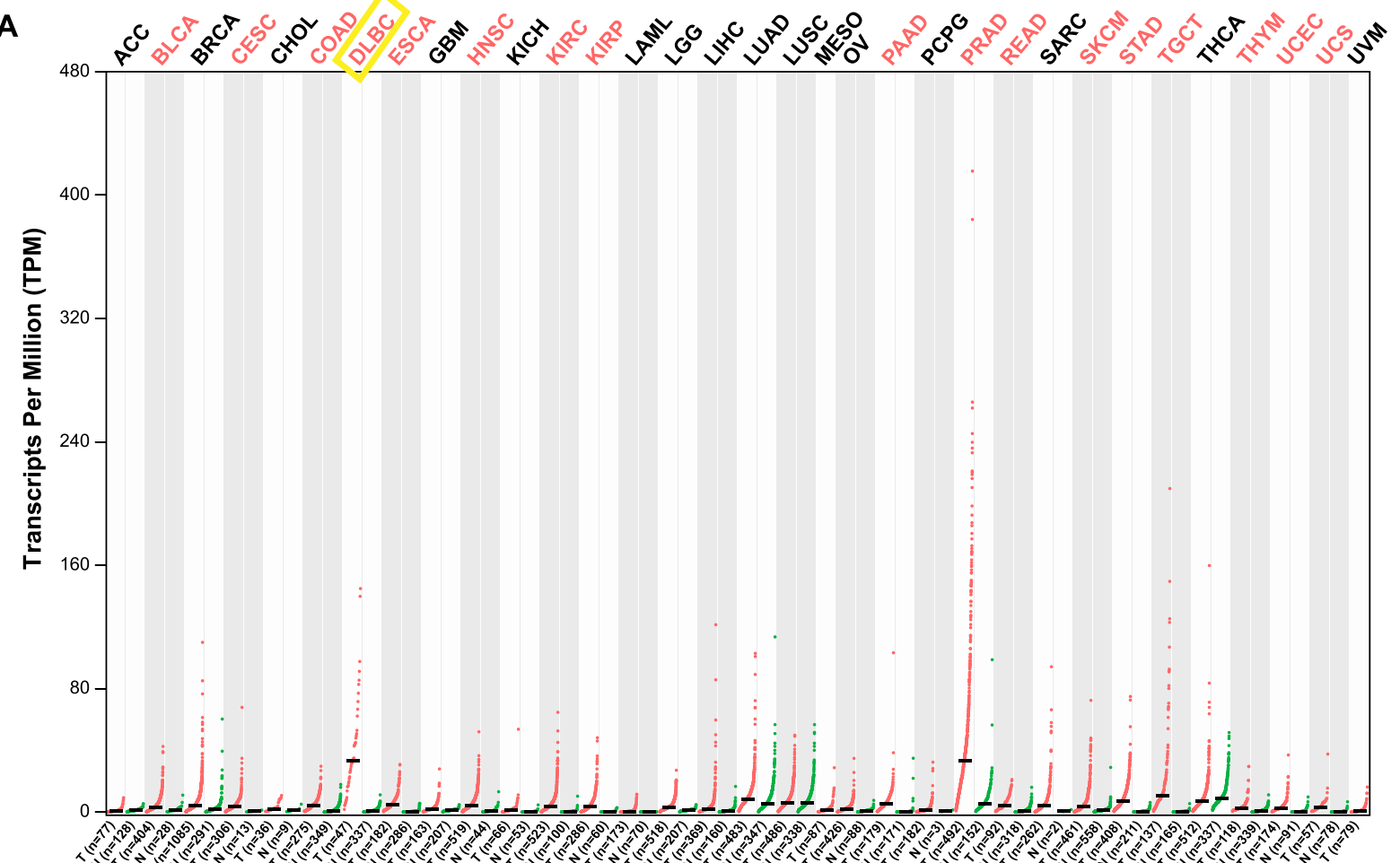

B

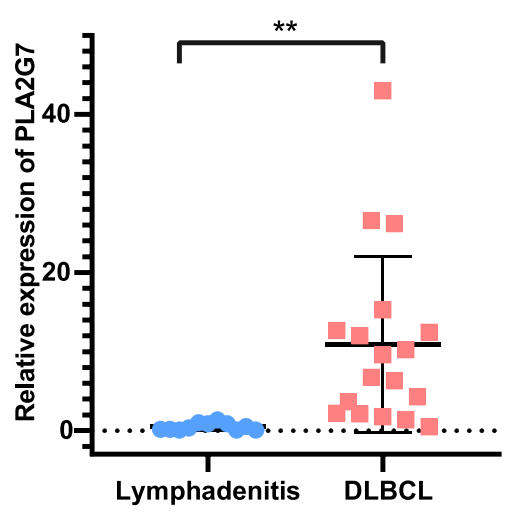

C

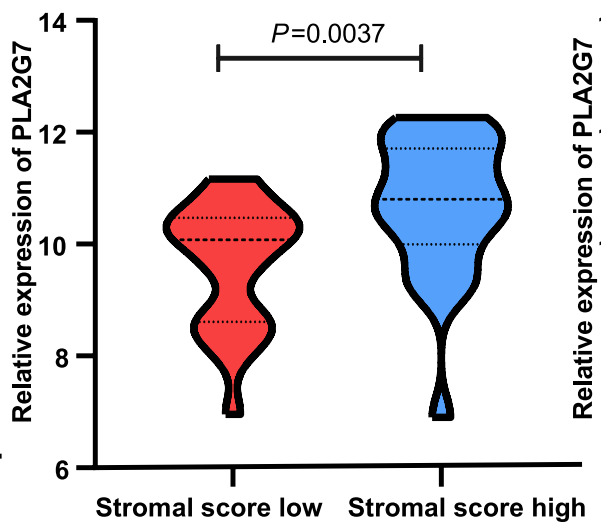

D

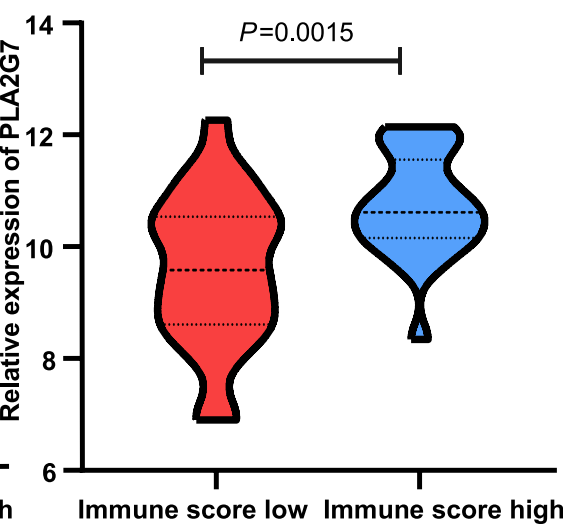

Fig. 5 The association between PLA2G7 and tumor progression. A: PLA2G7 overexpression was evident in 17 tumor tissue types, including DLBCL, relative to normal tissues as determined through GEPIA analyses. B: PLA2G7 expression in DLBCL patient samples and benign lymphadenitis tissue samples was compared. C and D: PLA2G7 expression in DLBCL samples with different stromal and immune scores was compared based upon the TCGA database

cells, and a milieu of cytokines and other compounds that play critical roles in modulating cancer onset and progression [24]. To fully explore the association between PLA2G7 expression and immune cell infiltration in the TME, CIBERSORT [25], which can be used to analyze the proportions of 22 of tumor-infiltrating immune cell (TIIC) types based upon RNA-seq data, was used to compare TIIC profiles in PLA2G7-low and -high DLBCL patients from the TCGA cohort (Fig. 6A). This analysis revealed that PLA2G7-high patients exhibited significantly higher proportions of monocytes and gamma delta $\mathrm{T}$ cells and lower frequencies of neutrophils relative to PLA2G7-low patients (Fig. 6B).

PLA2G7 promotes DLBCL cell proliferation and migration and inhibits apoptosis in vitro

To directly interrogate the role of PLA2G7 in DLBCL cells, we knocked down this gene in DB and SU-DHL-2 cell line and confirmed successful knockdown via qRTPCR using two different siRNA constructs (Fig. 7A). 
Table 1 Correlations between PLA2G7 and clinicopathological characteristics of DLBCL based on TCGA database

\begin{tabular}{|c|c|c|c|}
\hline \multirow[t]{2}{*}{ Characteristics } & \multicolumn{2}{|c|}{ PLA2G7 expression } & \multirow{2}{*}{$\begin{array}{l}P- \\
\text { value }\end{array}$} \\
\hline & High,no.cases & Low,no.cases & \\
\hline \multicolumn{4}{|l|}{ Age (years) } \\
\hline$\leq 57$ & 13 & 11 & \\
\hline$>57$ & 11 & 13 & 0.564 \\
\hline \multicolumn{4}{|l|}{ Gender } \\
\hline Female & 11 & 15 & \\
\hline Male & 13 & 9 & 0.247 \\
\hline \multicolumn{4}{|l|}{ Stromal score } \\
\hline Low & 8 & 16 & \\
\hline High & 16 & 8 & 0.021 \\
\hline \multicolumn{4}{|l|}{ Immune score } \\
\hline Low & 7 & 17 & \\
\hline High & 17 & 7 & 0.004 \\
\hline \multicolumn{4}{|c|}{ Maximum tumor dimension } \\
\hline NA & 6 & 4 & \\
\hline$\leq 3$ & 8 & 12 & \\
\hline$>3$ & 10 & 8 & 0.491 \\
\hline \multicolumn{4}{|c|}{ Primary therapy outcome } \\
\hline NA & 1 & 4 & \\
\hline$C R$ & 18 & 17 & \\
\hline$P R$ & 2 & 0 & \\
\hline SD & 1 & 1 & \\
\hline PD & 2 & 2 & 0.43 \\
\hline \multicolumn{4}{|l|}{ Tumor stage } \\
\hline NA & 2 & 4 & \\
\hline । & 4 & 4 & \\
\hline$\|$ & 9 & 8 & \\
\hline III & 4 & 1 & \\
\hline IV & 5 & 7 & 0.552 \\
\hline
\end{tabular}

Knockdown of PLA2G7 suppressed the ability of these DLBCL tumor cells to migrate and form colonies (Fig. $7 \mathrm{~B}$ and $\mathrm{C}$ ). Notably, such knockdown also markedly enhanced the death of these two DLBCL cells as measured in an Annexin V/7AA-D staining assay (Fig. 7D). Together, these data provide clear evidence in support of a model wherein PLA2G7 plays an oncogenic role in DLBCL by inhibiting tumor cell apoptotic death while simultaneously enhancing proliferation and migration.

Effect of darapladib on DLBCL proliferation and apoptosis To further explore the role of PLA2G7 as a therapeutic target, the cytotoxicity effect of darapladib, a specific inhibitor of PLA2G7, on DLBCL cells was investigated. DLBCL cells were treated with various concentrations of darapladib for $72 \mathrm{~h}$, and the cell proliferation ability was detected with a CCK8 assay. The results suggest that darapladib significantly attenuated the DB and SU-DHL2 cells viability, with IC50 values at $5.33 \mu \mathrm{M}$ and $12.92 \mu \mathrm{M}$, respectively (Fig. 8A). In addition, cell apoptosis analysis indicates darapladib resulted in a remarkable increase in the number of apoptotic cells when DB and SU-DHL-2 cells were treated with $5.33 \mu \mathrm{M}$ and $12.92 \mu \mathrm{M}$ darapladib for $48 \mathrm{~h}$, respectively (Fig. 8B). Consequently, darapladib could noticeably restrained the DLBCL cell viability and induced apoptosis.

\section{Clinical and prognostic significance of PLA2G7}

To further explore the clinical significance of PLA2G7, the relationship between PLA2G7 mRNA expression in peripheral blood and clinical characteristics of 53 DLBCL patients were analyzed (Table 2). The median PLA2G7 mRNA levels in DLBCL samples were regarded as the cut-off. High expression of PLA2G7 correlated with high levels of serum B2m $(P=0.0039)$, and low levels of serum albumin $(P=0.005)$ and $C D 10$ positive $(P=0.006)$. In contrast, low expression of PLA2G7 was found to be associated with the non-GCB subtype $(P=$ 0.034). Furthermore, survival analysis results indicate that DLBCL patients with high expression of PLA2G7 are associated with a worse prognosis (Fig. 8C).

\section{Discussion}

DLBCL is a common and highly heterogeneous form of NHL associated with high morbidity and mortality rates [26]. While roughly half of DLBCL cases can be cured via standard chemotherapy, treated relapsed or refractory DLBCL remains challenging [27]. It is thus essential that prognostic and diagnostic biomarkers of DLBCL be identified in order to guide patient detection and treatment. Herein, we therefore sought to identify potential prognostic and diagnostic biomarkers of DLBCL. In total, we identified 30 and 38 genes that were significantly up- and down-regulated, respectively, in DLBCL samples from two GEO datasets. Functional enrichment analyses suggested that these DEGs were associated with angiogenesis, cell proliferation, the immune response, and other key tumor progression-related pathways. Of these 68 DEGs, five were identified as promising diagnostic biomarkers of DLBCL through ROC curve analyses.

We next utilized the TCGA database in order to conduct WGCNA analyses exploring the relationship between gene expression profiles and DLBCL patient prognosis. Through this approach, we identified a key gene module that was related to patient outcomes. PLA2G7 was a hub gene within this module, and also exhibited favorable diagnostic utility in the above ROC curve analyses. Prior research indicates that PLA2G7 is a 


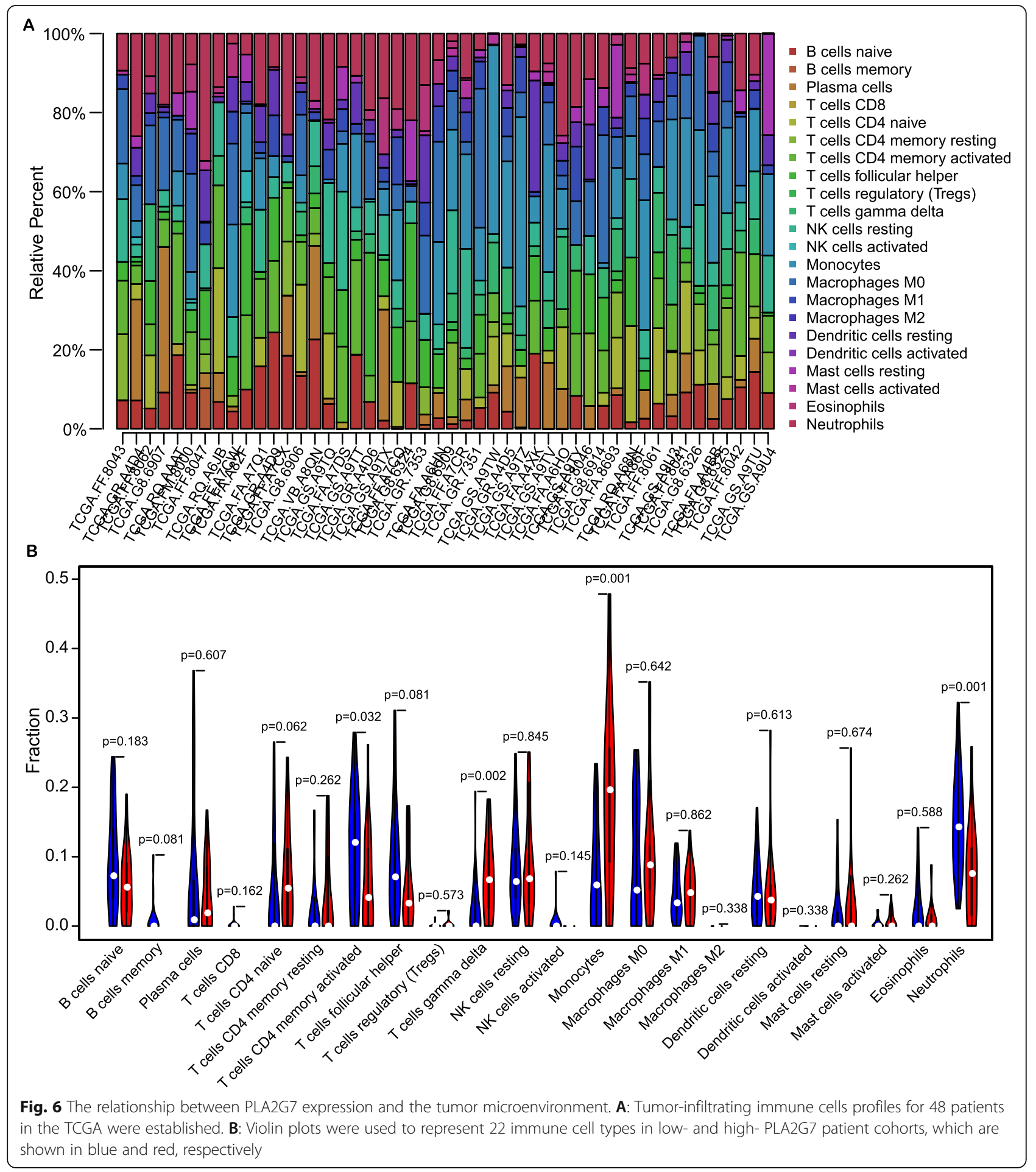

tumor-associated macrophage-derived factor that plays a key role in the regulation of tumor cell migration. In nasopharyngeal carcinoma (NPC) cells, there is evidence that PLA2G7 can enhance tumor cell migration and survival [28], and similar findings have also been observed in PCa cells [11]. Further research has led to the identification of this gene as a biomarker of primary and metastatic $\mathrm{PCa}$, and a viable therapeutic target in patients with ERG positive PCa [29]. In the GTex and TCGA databases, PLA2G7 was found to be upregulated in 17 different tumor types. Consistent with prior studies of PCa and NPC, we also determined that PLA2G7 promoted DLBCL cell proliferation and migration while suppressing the apoptotic death of these cells. 

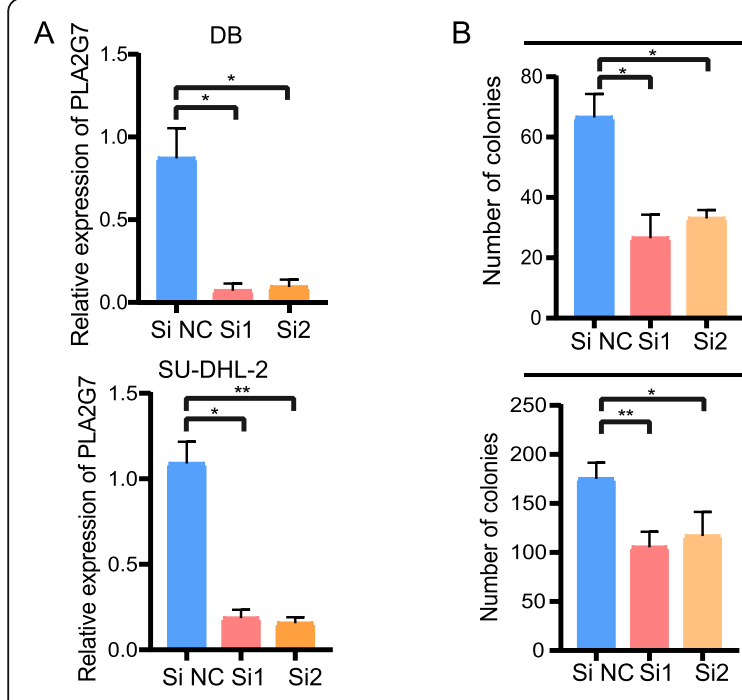

$\mathrm{DB}$
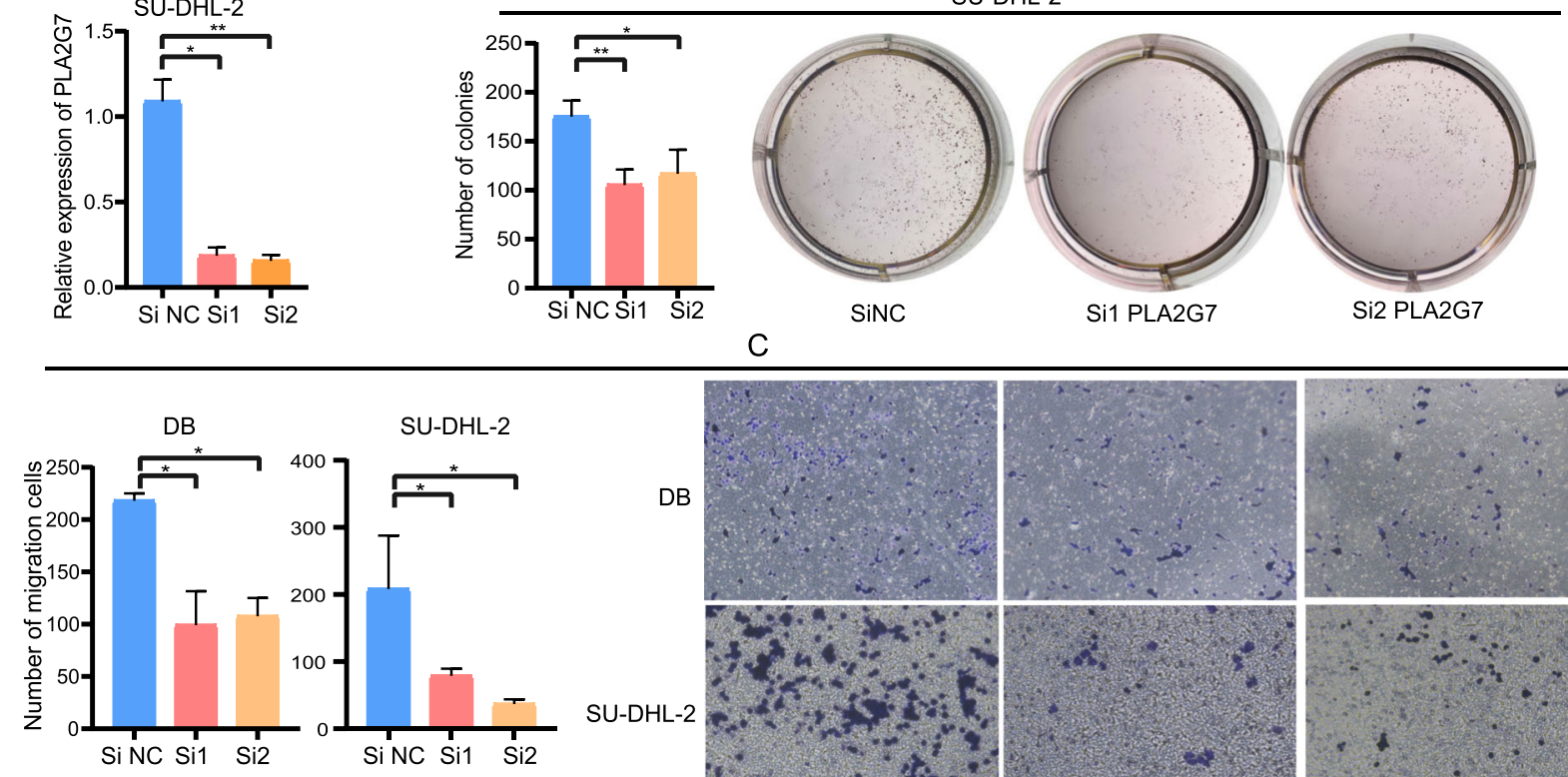

SU-DHL-2

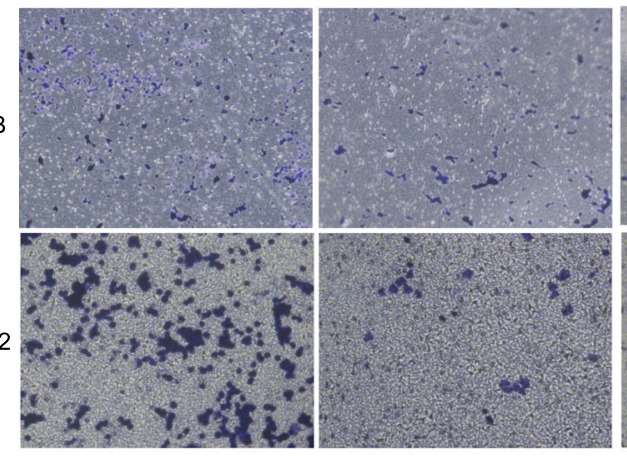

Si1 PLA2G7

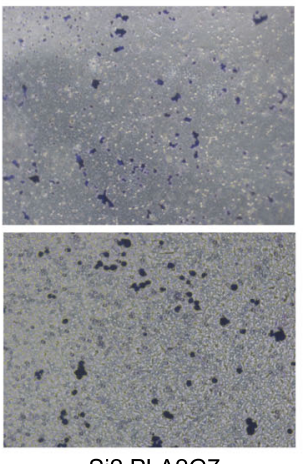

D
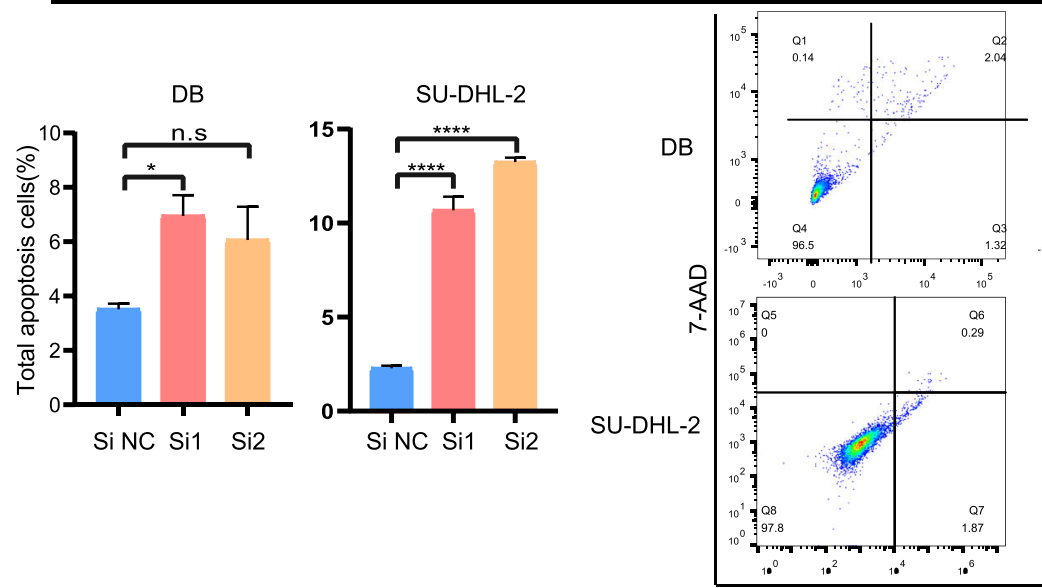

SiNC
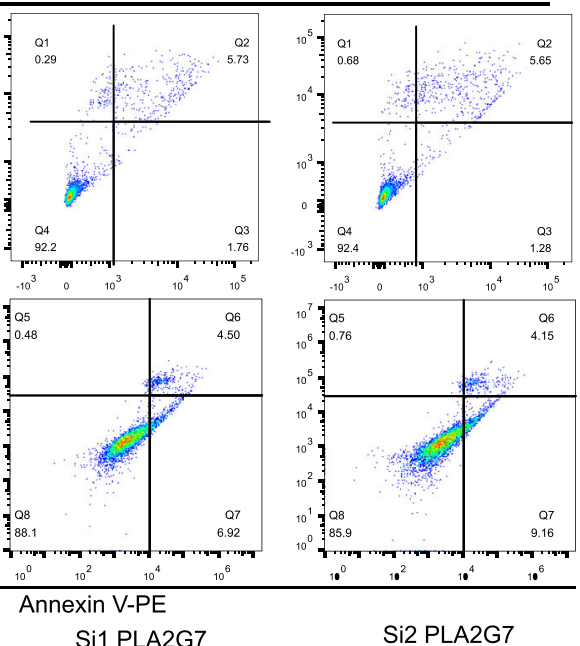

Fig. 7 PLA2G7 drives the in vitro migration and proliferation of DLBCL cells and inhibits their apoptotic death. A: PLA2G7 mRNA expression in DB and SU-DHL-2 cells following PLA2G7 siRNA transfection was assessed. B: Negative Control (NC) siRNA-, and PLA2G7 siRNA- transfected DB and SU-DHL-2 cells were evaluated in a colony formation assay. C: Cells prepared as in (B) were evaluated in a migration assay. D: Cells prepared as in (B) were assessed in an apoptosis assay. n.S, not significant, ${ }^{*} P \leq 0.05,{ }^{* *} P<0.01,{ }^{* *} P<0.001,{ }^{* * *} P<0.0001$ 


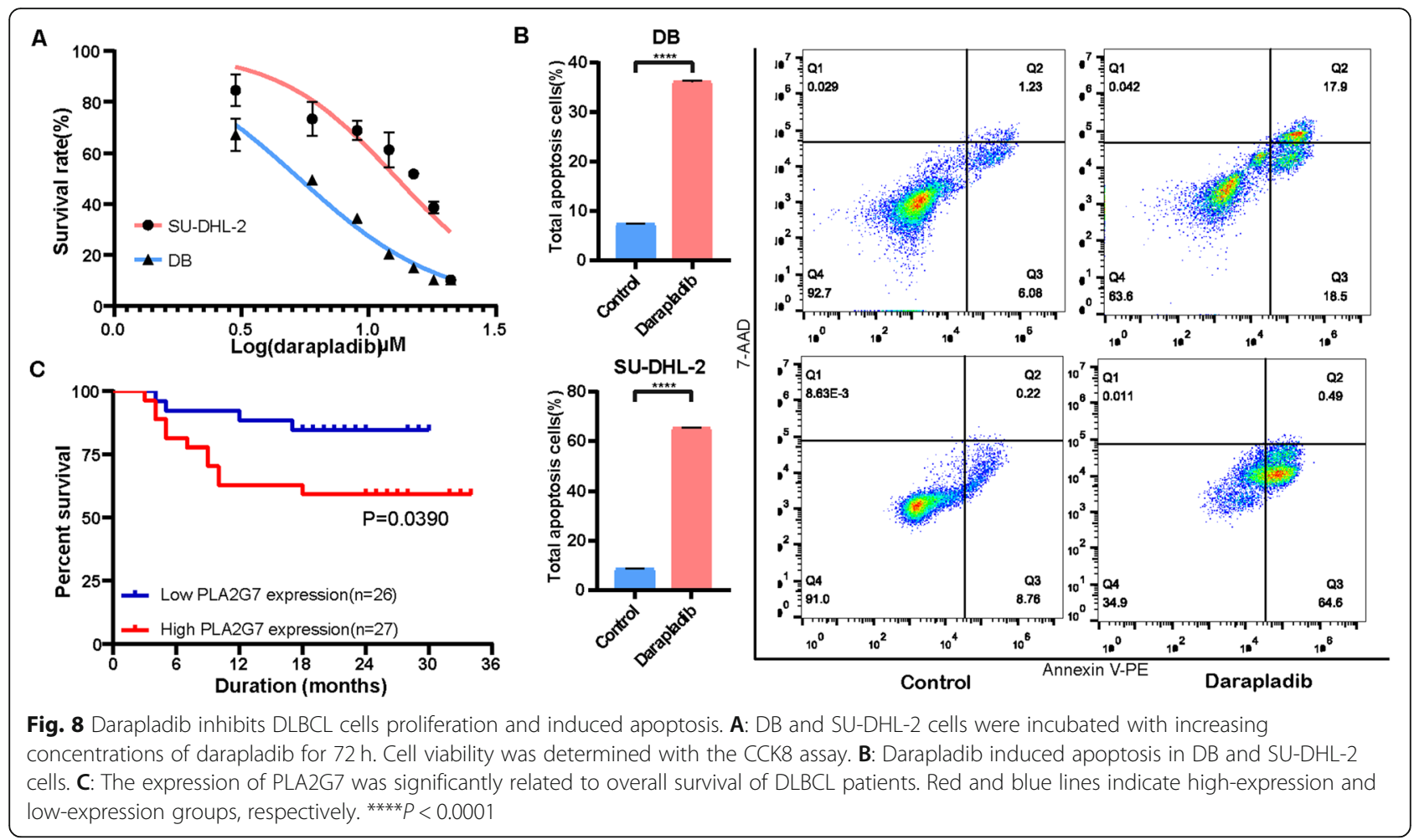

The tumor microenvironment includes macrophages, dendritic cells, $\mathrm{T}$ helper cells, $\mathrm{T}$ cytotoxic cells, and reactive B lymphocytes. Shain et al. [30] previously demonstrated that B cell tumor interactions with the local TME can influence tumor cell behavior by controlling the oncogenesis's growth and progression. Among these components, tumorassociated macrophages (TAM) were found to play a major role. The data generated in the present study further suggest that PLA2G7 expression may be associated with DLBCL tumor stromal and immune scores. Kua et al. [31, 32] reported that the CIBERSORT algorithm was used to analyze the DLBCL immune cell infiltration in the TME. As such, we explored the association between PLA2G7 expression and TIICs, revealing that patients expressing high levels of this gene also exhibited increased monocyte infiltration. Monocytes develop into macrophages in tumors, and are associated with poor prognosis in DLBCL patients due to IL-34 production [33]. It is reported that the OS and PFS of DLBCL patients with high expression of TAMs are often poor, and there is a positive correlation with the peripheral absolute monocyte count (AMC) [34]. Therefore, AMC is a useful prognostic marker that reflects the status of the tumor microenvironment (TME) in DLBCL. Increased tumor-infiltrating monocyte numbers may thus be responsible for the relationship between high PLA2G7 expression and poor DLBCL patient outcomes. Together, these data, therefore, offer insight into potential immunotherapeutic treatment strategies for this cancer type.

Notably, our study focused on the identification of PLA2G7 as a novel biomarker for DLBCL. However, it had some limitations. First, the sample size obtained from TCGA to validate the GEO data sets was not large, the data in TCGA about DLBCL lacked normal samples as controls and complete survival information was lacking in some cases. Future studies are required and should include a large sample size to validate such observations. Also, our study focused on bioinformatics methods and in vitro experiments to screen candidate genes for DLBCL and identified PLA2G7 as a novel biomarker, the functional role of PLA2G7 could be explored further to determine tumor cell migration using in vivo studies and a detailed mechanistic approach. Future studies are required and should include these approaches.

In summary, we herein identified PLA2G7 as a biomarker that is upregulated in DLBCL and that is related to the enhancement of DLBCL cell proliferation, invasion, and tumor microenvironmental composition. These findings suggest that PLA2G7 may not only be a 
Table 2 Correlations between PLA2G7 and clinicopathological characteristics of DLBCL peripheral blood. (GCB: germinal centre B-cell like; IPI: international prognostic index; CR: complete response; B2m: Recombinant Human beta-2-Microglobulin; LDH: lactate dehydrogenase; BCL: B-cell lymphoma)

\begin{tabular}{clll}
\hline Characteristics & PLA2G7 expression & $\begin{array}{l}\boldsymbol{P} \text { - } \\
\text { value }\end{array}$ \\
\cline { 2 - 3 } & High,no.cases $(\geq \mathbf{1 . 4 6 )}$ & Low,no.cases $(<\mathbf{1 . 4 6})$ & \\
\hline Age (years) & & 16 & 0.477 \\
$\quad \leq 54$ & 14 & 10 & \\
$>54$ & 13 & & 0.206
\end{tabular}

$\begin{array}{ccc}\text { Female } & 14 & 9 \\ \text { Male } & 13 & 17 \\ \text { stage } & & 10 \\ \text { I-II } & 5 & 16 \\ \text { III-IV } & 22 & \\ \text { Subtype } & & 7 \\ \text { GCB } & 13 & 19 \\ \text { Non-GCB } & 14 & \end{array}$

IPI score

$\begin{array}{lll}0-2 & 21 & 23 \\ >3 & 6 & 3\end{array}$

CR after first-line chemotheray

$\begin{array}{lll}\text { yes } & 14 & 17\end{array}$

no $13 \quad 9$

A/B symptoms

A 20

17

B $\quad 7$

Low serum albumin

yes

\section{1}

no

16

High serum B2m

$\begin{array}{ll}\text { yes } & 19 \\ \text { no } & 8\end{array}$

High serum LDH

$\begin{array}{lll}\text { yes } & 15 & 1 \\ \text { no } & 12 & 15\end{array}$

CD10

$\begin{array}{ccc}\text { positive } & 15 & 5 \\ \text { negative } & 12 & 21 \\ \text { CD20 } & & \\ \text { positive } & 26 & 25 \\ \text { negative } & 1 & 1\end{array}$

BCL-2

$\begin{array}{lll}\text { positive } & 25 & 22 \\ \text { negative } & 2 & 4\end{array}$

BCL-6
0.107 0.005

Table 2 Correlations between PLA2G7 and clinicopathological characteristics of DLBCL peripheral blood. (GCB: germinal centre B-cell like; IPI: international prognostic index; CR: complete response; B2m: Recombinant Human beta-2-Microglobulin; LDH: lactate dehydrogenase; BCL: B-cell lymphoma) (Continued)

\begin{tabular}{|c|c|c|c|}
\hline \multirow[t]{2}{*}{ Characteristics } & \multicolumn{2}{|l|}{ PLA2G7 expression } & \multirow{2}{*}{$\begin{array}{l}P \text { - } \\
\text { value }\end{array}$} \\
\hline & High,no.cases( $\geq 1.46)$ & Low,no.cases $(<1.46)$ & \\
\hline positive & 24 & 19 & \\
\hline negative & 3 & 7 & \\
\hline
\end{tabular}

diagnostic and prognostic biomarker of DLBCL, but also a viable therapeutic target for the improvement of patient outcomes.

\section{Abbreviations}

DLBCL: Diffuse large B-cell lymphoma; GEO: Gene expression omnibus; WCGNA: Weighted gene co-expression network; TCGA: The cancer genome atlas; DEG: Differentially expressed gene; GO: Gene ontology; KEGG: Kyoto encyclopedia of genes and genomes; ROC: Receiver operating characteristic; RRA: RobustRankAggreg

\section{Supplementary Information}

The online version contains supplementary material available at https:/doi. org/10.1186/s12885-021-08660-4.

\section{Additional file 1.}

Additional file 2.

\section{Acknowledgements}

We thank Professor Haiying Fu and Professor Hurong Zhou for offering expert advice concerning this study.

\section{Authors' contributions}

ZW: conceptualization, methodology, investigation, original draft, review and

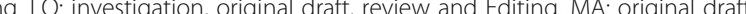
review and Editing. LZ: methodology, original draft, review and Editing. SJ: funding acquisition and supervision. All authors read and approved the final version of the manuscript.

\subsection{Funding}

This study was supported in part by Joints Funds for the Innovation of Science and Technology, Fujian Province (2018Y9205); Qihang Foundation of Fujian Medical University (2020QH2015); Construction project of Fujian. medical center of hematology (Min201704) and sponsored by National and Fujian Provincial Key Clinical Specialty Discipline Construction Program, P. R.C.

\section{Availability of data and materials}

0.006 The datasets used or analysed during the current study are available from the corresponding author on reasonable request.

\section{Declarations}

Ethics approval and consent to participate

The study was approved by the Ethics Committee of Fujian Medical University Union Hospital. All experiments were performed according to the relevant regulations and written informed consent was obtained from patients.

\section{Consent for publication}

Not applicable.

\section{Competing interests}

The authors declare that they have no competing interests. 


\section{Author details}

${ }^{1}$ Fujian Institute of Hematology, Fujian Medical Center of Hematology, Fujian Provincial Key Laboratory on Hematology; Fujian Medical University Union Hospital, Fuzhou, China. ${ }^{2}$ Meng Chao Hepatobiliary Hospital Affiliated to Fujian Medical University, Fuzhou, China.

Received: 6 March 2021 Accepted: 1 August 2021

Published online: 17 August 2021

\section{References}

1. Swerdlow S, Campo E, Pileri S, Harris N, Stein H, Siebert R, et al. The 2016 revision of the World Health Organization classification of lymphoid neoplasms. Blood. 2016;127(20):2375-90. https://doi.org/10.1182/blood-201 6-01-643569.

2. Shree T, Li Q, Glaser S, Brunson A, Maecker H, Haile R, et al. Impaired immune health in survivors of diffuse large B-cell lymphoma. J Clin Oncol. 2020;38(15):1664-75. https://doi.org/10.1200/JCO.19.01937.

3. Li M, Chiang Y, Lyssiotis C, Teater M, Hong J, Shen H, et al. Non-oncogene Addiction to SIRT3 Plays a Critical Role in Lymphomagenesis. Cancer Cell. 2019;35(6):916-931.e919.

4. Deng W, Clipson A, Liu H, Huang Y, Dobson R, Wang M, et al. Variable responses of MYC translocation positive lymphoma cell lines to different combinations of novel agents: impact of BCL2 family protein expression. Transl Oncol. 2018;11(5):1147-54. https://doi.org/10.1016/j.tranon.2018.07. 007.

5. Reddy A, Zhang J, Davis N, Moffitt A, Love C, Waldrop A, et al. Genetic and functional drivers of diffuse large B cell lymphoma. Cell. 2017;171(2):481494.e415.

6. Su W, Niu X, Ji H, Xu Y, Zhong L, Wang S, et al. A novel classification based on B-cell receptor signal gene expression correlates with prognosis in primary breast diffuse large B-cell lymphoma. J Cancer. 2020;11(9):2431-41. https://doi.org/10.7150/jca.39083.

7. Shukla A, Tripathi D. Detecting biomarkers from microarray data using distributed correlation based gene selection. Genes Genomics. 2020;42(4): 449-65. https://doi.org/10.1007/s13258-020-00916-w.

8. Sun C, Cheng X, Wang C, Wang X, Xia B, Zhang Y. Gene expression profiles analysis identifies a novel two-gene signature to predict overall survival in diffuse large B-cell lymphoma. Biosci Rep. 2019;3;39(1):BSR20181293. https:// doi.org/10.1042/BSR20181293.

9. Suh K, Sarojini S, Youssif M, Nalley K, Milinovikj N, Elloumi F, et al. Tissue banking, bioinformatics, and electronic medical records: the front-end requirements for personalized medicine. J Oncol. 2013;2013:368751.

10. Liu X, Wu J, Zhang D, Bing Z, Tian J, Ni M, et al. Identification of potential key genes associated with the pathogenesis and prognosis of gastric Cancer based on integrated bioinformatics analysis. Front Genet. 2018;9:265. https://doi.org/10.3389/fgene.2018.00265.

11. Vainio P, Lehtinen L, Mirtti T, Hilvo M, Seppänen-Laakso T, Virtanen J, et al. Phospholipase PLA2G7, associated with aggressive prostate cancer, promotes prostate cancer cell migration and invasion and is inhibited by statins. Oncotarget. 2011;2(12):1176-90. https://doi.org/10.18632/oncota rget.397.

12. Alinezhad S, Väänänen R, Mattsson J, Li Y, Tallgrén T, Tong Ochoa N, et al. Validation of novel biomarkers for prostate Cancer progression by the combination of bioinformatics. clinical and functional studies. PloS One. 2016:11(5):e0155901. https://doi.org/10.1371/journal.pone.0155901.

13. Saenger $Y$, Magidson J, Liaw B, de Moll E, Harcharik S, Fu Y, et al. Blood mRNA expression profiling predicts survival in patients treated with tremelimumab. Clin Cancer Res. 2014;20(12):3310-8. https://doi.org/10.11 58/1078-0432.CCR-13-2906.

14. Gómez-Abad C, Pisonero H, Blanco-Aparicio C, Roncador G, GonzálezMenchén A, Martinez-Climent J, et al. PIM2 inhibition as a rational therapeutic approach in B-cell lymphoma. Blood. 2011;118(20):5517-27. https://doi.org/10.1182/blood-2011-03-344374

15. Dybkær K, Bøgsted M, Falgreen S, Bødker J, Kjeldsen M, Schmitz A, et al. Diffuse large B-cell lymphoma classification system that associates normal Bcell subset phenotypes with prognosis. J Clin Oncol. 2015;33(12):1379-88. https://doi.org/10.1200/JCO.2014.57.7080.

16. Yoshihara K, Shahmoradgoli M, Martínez E, Vegesna R, Kim H, Torres-Garcia W, et al. Inferring tumour purity and stromal and immune cell admixture from expression data. Nat Commun. 2013;4(1):2612. https://doi.org/10.1038/ ncomms3612.
17. Zhou Y, Chen L, Zhang Y, Hu S, Dong Z, Wu M, et al. Integrated transcriptomic analysis reveals hub genes involved in diagnosis and prognosis of pancreatic cancer. Mol Med. 2019;25(1):47.

18. Kanehisa M, Goto S. KEGG: Kyoto encyclopedia of genes and genomes. Nucleic Acids Res. 2000;28(1):27-30. https://doi.org/10.1093/nar/28.1.27.

19. Kanehisa M. Toward understanding the origin and evolution of cellular organisms. Protein Sci. 2019;28(11):1947-51. https://doi.org/10.1002/pro.371 5.

20. Kanehisa M, Furumichi M, Sato Y, Ishiguro-Watanabe M, Tanabe M. KEGG: integrating viruses and cellular organisms. Nucleic Acids Res. 2021;49(D1): D545-51. https://doi.org/10.1093/nar/gkaa970.

21. Langfelder P, Horvath S. Fast R Functions for Robust Correlations and Hierarchical Clustering. J Stat Softw. 2012;46(11):i11. https://doi.org/10.1863 7/jss.v046.i11.

22. Tang J, Kong D, Cui Q, Wang K, Zhang D, Gong Y, et al. Prognostic genes of breast Cancer identified by gene co-expression network analysis. Front Oncol. 2018;8:374. https://doi.org/10.3389/fonc.2018.00374

23. Tang Z, Li C, Kang B, Gao G, Li C, Zhang Z. GEPIA: a web server for cancer and normal gene expression profiling and interactive analyses. Nucleic Acids Res. 2017;45(W1):W98-W102. https://doi.org/10.1093/nar/gkx247.

24. Luo $Y$, Zeng G, Wu S. Identification of microenvironment-related prognostic genes in bladder Cancer based on gene expression profile. Front Genet. 2019;10:1187. https://doi.org/10.3389/fgene.2019.01187.

25. Newman A, Liu C, Green M, Gentles A, Feng W, Xu Y, et al. Robust enumeration of cell subsets from tissue expression profiles. Nat Methods. 2015:12(5):453-7. https://doi.org/10.1038/nmeth.3337.

26. Martelli M, Ferreri A, Agostinelli C, Di Rocco A, Pfreundschuh M, Pileri S. Diffuse large B-cell lymphoma. Crit Rev Oncol Hematol. 2013;87(2):146-71. https://doi.org/10.1016/j.critrevonc.2012.12.009.

27. Wang X, Tan Y, Huang Z, Huang N, Gao M, Zhou F, et al. Disrupting myddosome assembly in diffuse large B-cell lymphoma cells using the MYD88 dimerization inhibitor ST2825. Oncol Rep. 2019;42(5):1755-66. https://doi.org/10.3892/or.2019.7282.

28. Low H, Png C, Li C, Wang Y, Wong S, Zhang Y. Monocyte-derived factors including PLA2G7 induced by macrophage-nasopharyngeal carcinoma cell interaction promote tumor cell invasiveness. Oncotarget. 2016;7(34):5547390. https://doi.org/10.18632/oncotarget.10980.

29. Vainio P, Gupta S, Ketola K, Mirtti T, Mpindi J, Kohonen P, et al. Arachidonic acid pathway members PLA2G7, HPGD, EPHX2, and CYP4F8 identified as putative novel therapeutic targets in prostate cancer. Am J Pathol. 2011; 178(2):525-36. https://doi.org/10.1016/j.ajpath.2010.10.002.

30. Shain $\mathrm{K}$, Dalton $\mathrm{W}$, Tao J. The tumor microenvironment shapes hallmarks of mature B-cell malignancies. Oncogene. 2015;34(36):4673-82. https://doi. org/10.1038/onc.2014.403.

31. Kuang Z, Li X, Liu R, Chen S, Tu J. Comprehensive characterization of Cachexia-inducing factors in diffuse large B-cell lymphoma reveals a molecular subtype and a prognosis-related signature. Front Cell Dev Biol. 2021:9:648856. https://doi.org/10.3389/fcell.2021.648856.

32. Qiao L, Li H, Zhang Y, Shen D, Liu P, Che Y. CD24 contributes to treatment effect in $\mathrm{ABC}-\mathrm{DLBCL}$ patients with $\mathrm{R}-\mathrm{CHOP}$ resistance. Pharmacogenomics Personalized Med. 2021;14:591-9. https://doi.org/10.2147/PGPM.S310816.

33. Noyori $\mathrm{O}$, Komohara $\mathrm{Y}$, Nasser $\mathrm{H}$, Hiyoshi M, Ma C, Pan C, et al. Expression of IL-34 correlates with macrophage infiltration and prognosis of diffuse large B-cell lymphoma. Clin Transl Immunol. 2019;8(8):e1074. https://doi.org/10.1 002/cti2.1074.

34. Li Y, Shi Z, Wang X, Gu K, Zhai Z. Tumor-associated macrophages predict prognosis in diffuse large B-cell lymphoma and correlation with peripheral absolute monocyte count. BMC Cancer. 2019;19(1):1049. https://doi.org/1 $0.1186 / s 12885-019-6208-x$

\section{Publisher's Note}

Springer Nature remains neutral with regard to jurisdictional claims in published maps and institutional affiliations. 